GROOVE PANCREATITIS MASQUERADING AS CARCINOMA PANCREAS : A DIAGNOSTIC DILEMMA.

\title{
Hepatobiliary Surgery
}

Lohith P*

MBBS, MS(General Surgery), Senior Resident (Registrar) in Department of General Surgery, Maulana Azad Medical College and associated Loknayak Hospital, New Delhi110002, India. *Corresponding Author

\section{Rajshekar P}

MBBS, MS Postgraduate resident in Department of General Surgery, Maulana Azad

Deepak Ghuliani

Medical College and associated Loknayak Hospital, New Delhi-110002, India.

MBBS, MS(General Surgery), Professor in Department of General surgery, Maulana Azad Medical College and associated Loknayak Hospital, New Delhi-110002, India.

\section{Ravindra K Saran}

MBBS, MD(Pathology), Director Professor in Department of Pathology, GIPMER, New Delhi-110002, India.

\section{ABSTRACT}

INTRODUCTION: Groove pancreatitis(GP) is a rare special form of chronic pancreatitis localised to pancreaticoduodenal groove, presents commonly with signs and symptoms of duodenal obstruction, mimicks pancreatic cancer radiologically and the surgeon proceeds with inadvertent whipples procedure.

PRESENTATION OF CASE: A28yr old gentleman, alcoholic presented with duodenal obstruction for 3days. CECT abdomen was suggestive of exophytic lesion from second part of duodenum ?duodenal diverticulum. UGIE showed large growth with overlying abnormal mucosa causing luminal compromise in second part of duodenum. Biopsy was taken which showed normal villous pattern. Patient was managed conservatively and improved gradually. On further evaluation, EUS showed $5 \times 5.5 \mathrm{~cm}$ cystic space occupying lesion in close relation to second part of duodenum and head of pancreas ?origin. EUS guided FNAC showed features suggestive of adenocarcinoma. With this pathological diagnosis, patient was taken up for Whipple's procedure, intraoperatively, $3 \mathrm{~cm}$ mass lesion was noted in the pancreaticoduodenal groove. HPE of the specimen showed a haemorraghic nodule $(3 \times 1.8 \times 1.2 \mathrm{~cm})$ in the duodenal wall and changes of chronic pancreatitis in the pancreticoduodenal groove suggestive of GP. Postoperative period and follow up of 6 months was uneventful.

DISCUSSION: In GP, EUS guided FNAC may reveal large gaint cells, spindle cells or hyperplasia of brunner glands depending on the area of sampling and these features mimic neoplasia as observed in our case. MRI criteria given by Kalb et al show diagnostic accuracy of $87.2 \%$ for GP and negative predictive value of $92.9 \%$ to rule out pancreatic cancer. Arvanitakis et al showed stepwise management approach is effective in GP and with combination of medical and endoscopic treatment, complete clinical response rate was observed in $80 \%$.

CONCLUSION: It is important to diagnose and differentiate GP from pancreatic cancer preoperatively and avoid morbidity from unnecessary pancreaticoduodenectomy in patients of GP.

\section{KEYWORDS}

groove pancreatitis; whipples procedure; pancreaticoduodenal groove.

\section{INTRODUCTION:}

Groove pancreatitis is a special rare form of chronic pancreatitis which is localised to pancreaticoduodenal groove, the region bounded by head of pancreas, duodenum and common bile duct. The term was first described by stolte et al in $1982^{1}$. It presents most commonly with signs and symptoms of duodenal obstruction, mimicks pancreatic cancer radiologically and the surgeon proceeds with inadvertent whipples procedure. Here we report a similar case of groove pancreatitis in line with SCARE criteria

\section{PRESENTATION OFACASE:}

A 28yr old male patient, chronic alcoholic, with no past history of biliary or gastric surgery presented with postprandial pain and distension of upper abdomen with bilious vomiting for 3 days. Physical examination was unremarkable except for the presence of succussion splash. X ray abdomen, supine and erect view were inconclusive. USG abdomen showed heterogenous lesion measuring $5 \times 7 \mathrm{~cm}$ in hepatoduodenal pouch in relation to the second part of Duodenum. CECT abdomen showed a relatively well defined heterogeneously enhancing exophytic hypodense lesion measuring $7.2 \times 5.4 \times 4.5 \mathrm{~cm}$ seen arising from second and third part of duodenum with cystic areas within abutting head of pancreas medially ?Duodenal diverticulum(Fig 1).

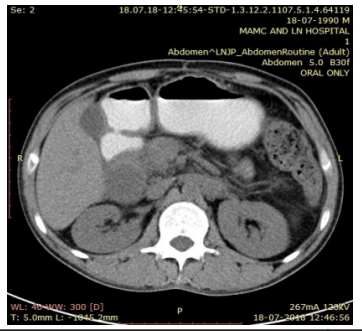

Submitted :
Serum amylase, lipase levels and tumour marker(Ca 19-9) were normal. UGIE showed large growth with overlying abnormal mucosa causing luminal compromise in second part of duodenum. Scope was not negotiable beyond. ?Malignant infiltration of second part of duodenum. Biopsy was taken and showed normal villous pattern. Patient was managed conservatively with nil by mouth, nasogastric aspiration and analgesics. Patient improved gradually and was started orally, accepted semisolid diet. On further evaluation, EUS showed $5 \times 5.5 \mathrm{~cm}$ cystic space occupying lesion in close relation to second part of duodenum and head of pancreas ?origin. EUS guided FNAC showed features suggestive of adenocarcinoma. With this pathological diagnosis, patient was taken up for Whipple's procedure, intraoperatively a mass lesion of around $3 \mathrm{~cm}$ was noted in the pancreaticoduodenal groove. Histopathological examination of the specimen showed a haemorraghic nodule $(3 \times 1.8 \times 1.2 \mathrm{~cm})$ in the duodenal wall and changes of chronic pancreatitis in the pancreticoduodenal groove suggestive of groove pancreatitis (Fig 2).
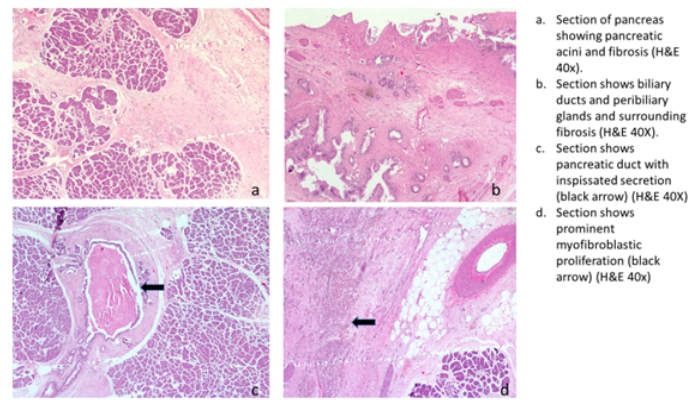

Postoperative period was uneventful. The patient is doing well after 6 months follow up.

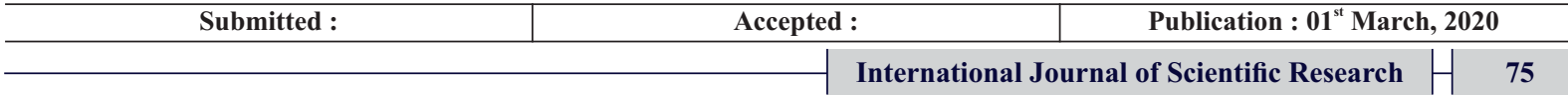


DISCUSSION:

Groove pancreatitis has been addressed with multiple names like cystic dystrophy of heterotopic pancreas, myoadenomatosis, paraduodenal wall cyst, paraduodenal pancreatitis. The exact incidence is not known, it varies from 2.7 to $24.5 \%$ cases of whipples procedure done for chronic pancreatitis ${ }^{1,3}$. It can be of two types, Pure and Segmental form as described by Becker et $\mathrm{al}^{3}$ in 1991. Pure form involves the pancreaticoduodenal groove only where as Segmental form involves head of pancreas with stenosis of pancreatic duct in addition to the groove.

Etiopathogenesis still remains unclear. One of the most common mechanisms described in the literature is altered pancreatic secretion in duct of Santorini ${ }^{4-6}$. When the duct of Santorini's outflow is blocked by impacted protein plugs due to unknown reasons, flow is directed towards the pancreatic body where duct of Santorini joins the main pancreatic duct at an acute angle. This interferes in flow of protein rich and more viscous pancreatic juice (consequence of chronic consumption of alcohol) with resultant accumulation of secretion in the cranial part of the pancreatic head and leakage into pancreaticoduodenal groove results in groove pancreatitis? ${ }^{7}$ Other probable causes may be Brunner's gland hyperplasia, ectopic rest of pancreas, duodenal bud, fibrous scarring secondary to biliary disease, gastrectomy, gastroduodenal ulcer ${ }^{8,9}$. Our patient had a significant history of chronic alcoholic intake.

Patients of groove pancreatitis present classically with postprandial pain, early satiety, vomiting and weight loss because of duodenal obstruction and usually will be middle aged men with chronic history of alcohol intake as observed in our case'. The presentation may be acute or chronic. Jaundice may be present if there is stenosis of distal CBD but occurs rarely.

On evaluation, groove pancreatitis tends to mimic pancreatic cancer radiologically and its important to be aware of significant differences between the two. On CT, the pure form will have findings of heterogenously enhancing hypodense plate like lesion in the pancreaticoduodenal groove and thickened duodenal wall with multiple cysts ${ }^{5}$. Similar findings was observed in our case. The segmental form will have findings of hypodense focal lesion in head of pancreas close to duodenum and there may be constriction of distal CBD. It is very important to note that even in extensive disease there will be features of infiltration of peripancreatic vessels ${ }^{5}$. EUS may help in diagnosis but insertion of scope may not be possible always due to duodenal stenosis and EUS guided fine needle aspiration may reveal large gaint cells, spindle cells or hyperplasia of brunner glands depending on the area of sampling and these features mimic neoplasia as observed in our case. Also an area of fibrosis cannot rule out neoplasia because it may occur due to desmoplastic reaction associated with neoplasia ${ }^{9}$.

Recently, a MRI criteria with three strict findings was given by Kalb et $\mathrm{al}^{10}$ with a diagnostic accuracy of $87.2 \%$ for groove pancreatitis and negative predictive value of $92.9 \%$ to rule out pancreatic cancer. It included cystic lesions in the region of accessory pancreatic duct, focal thickening in the second part of duodenum and an abnormally increased enhancement in second part of duodenum.

Management of Groove pancreatitis includes conservative management with abstinence from alcohol, analgesics and bowel rest, endoscopically with stenting of minor papilla in the short term. Some may eventually need surgical management with pancreaticoduodenectomy procedure. Arvanitakis et $\mathrm{al}^{11}$ showed that stepwise management approach is effective and with a combination of medical and endoscopic treatment, complete clinical response rate was observed to be $80 \%$.

In our case, the patient had improved with conservative management for the initial presentation and was asymptomatic at the time of further evaluation and preoperative period. But as there was a diagnostic dilemma and EUS guided FNAC was suggestive of adenocarcinoma we proceeded with pancreaticoduodenectomy.

\section{CONCLUSION:}

It is important to diagnose and differentiate groove pancreatitis from pancreatic cancer whenever possible preoperatively and avoid overtreating the patient and morbidity from unnecessary pancreaticoduodenectomy in patients of Groove pancreatitis.
REFERENCES:

1. Stolte M, Weiss W, Volkholz H, Rosch W. A special form of segmental pancreatitis: "groove pancreatitis". Hepatogastroenterology. 1982;29:198-208.

2. Agha RA, Borrelli MR, Farwana R, Koshy K, Fowler A, Orgill DP, For the SCARE Group. The SCARE 2018 Statement: Updating Consensus Surgical CAse REpor (SCARE) Guidelines, International Journal of Surgery 2018;60:132-136Becker V, Mischke U. Groove pancreatitis. Int J Pancreatol. 1991;10:173-82.

3. Ferreira A, Remalho M, Herédia V, et al. Groove pancreatitis: a case report and review of the literature. J Radiol Case Rep. 2010;4:9-17.

4. Triantopoulou C, Dervenis C, Giannakou N, Papailiou J, Prassopoulos P. Groove pancreatitis: a diagnostic challenge. Eur Radiol. 2009;19(7):1736-1743.

5. Kloppel G. Chronic pancreatitis, pseudotumors and other tumor-like lesions. Mod Pathol. 2007;20(Supl. 1):S113-S131.

6. Shudo R, Yazaki Y, Sakurai S, Uenishi H, Yamada H, Sugawara K, et al. Groove pancreatitis: Report of a case and review of the clinical and radiologic features of groove pancreatitis reported in Japan. Intern Med. 2002;41:537-42.

7. Zamboni G, Capelli P, Scarpa A, et al. Nonneoplastic mimickers of pancreatic neoplasms. Arch Pathol Lab Med. 2009;133(3):439-453

8. Irie H, Honda $\mathrm{H}$, Kuroiwa T, et al. MRI of groove pancreatitis. J Comput Assist Tomogr. 1998:22:651-655.

9. Ray S, Ghatak S, Mishra D, et al. Groove pancreatitis: Report of three cases with brief review of literature. Indian J Surg. 2017;79(4):344-348

10. Kalb B, Martin DR, Sarmiento JM, et al. Paraduodenal pancreatitis: clinica performance of MR imaging in distinguishing from carcinoma. Radiology. 2013;269:475-481.

11. Arvanitakis M, Rigaux J, Toussaint E, et al. Endotherapy for paraduodenal pancreatitis: a large retrospective case series. Endoscopy. 2014;46:580-587. 